\title{
What trends in non-classical logic were anticipated by Nikolai Vasiliev?
}

\author{
VLADIMIR I. MARKIN
}

\begin{abstract}
In this paper we discuss a question about the trends in non-classical logic that were exactly anticipated by Nikolai Vasiliev. We show the influence of Vasiliev's Imaginary logic on paraconsistent logic. Metatheoretical relations between Vasiliev's logical systems and many-valued predicate logics are established. We also make clear that Vasiliev has developed a sketch of original system of intensional logic and expressed certain ideas of modal and temporal logics.
\end{abstract}

Keywords: Nikolai Vasiliev, imaginary logic, syllogistic, paraconsistent logic, many-valued logic, intensional logic, modal logic, temporal logic

A century has passed since the publication of logical works of outstanding Russian logician Nikolai Vasiliev. Now he is rightfully considered to be the founder of non-classical logic together with Łukasiewicz, Lewis, and Brouwer. Vasiliev has published only three papers on logic between 1910 and 1913, but these papers gave long lasting effect on the development of non-classical logic in 20-th century.

There are various opinions on the subject of what trends in Nonclassical logic were exactly anticipated by Nikolai Vasiliev.

Vasiliev is ordinary regarded to be the forerunner of paraconsistent logic. It is true that Vasiliev's idea to construct a consistent logic of contradictory world (Imaginary, non-Aristotelian logic) is really in keeping with some informal attitudes of paraconsistent logic.

Before paraconsistent logic was set up, some researchers considered Vasiliev's works to contain the ideas of many-valued logic. The reason for this opinion was that Vasiliev introduced the third form of 
propositions ('indifferent', or contradictory statements) along with affirmative and negative statements. Besides, Vasiliev put forward an idea of logic of $n$ dimensions which has $n$ initial qualities of propositions.

We'll also make clear that Vasiliev has developed a sketch of original system of intensional logic and expressed certain ideas of modal and temporal logics.

\section{Imaginary logic: consistent logic of contradictory world}

The best known Vasiliev's logical system is his Imaginary nonAristotelian logic - one of the first ever non-classical logical theories.

Inspired by the ideas of non-Euclidean geometry contributed by his colleague from Kazan University Nilolai Lobachevski, Vasiliev in his paper 'Imaginary (non-Aristotelian) logic' [1, pp. 53-93] constructed a deductive theory of syllogistic kind. The language of this theory contains besides affirmative and negative propositions contradictory (so called indifferent) ones with syllogistic copula 'is and is not simultaneously'. According to Vasiliev, such propositions are false in our terrestrial world but can turn to be true in a certain imaginary world.

A. Arruda was the first who appreciated Vasiliev as a forerunner of paraconsistent logic. On the basis of Vasiliev's ideas she formulated three propositional calculi V1-V3 useful as a logical part of non-trivial inconsistent theories [7].

V3 calculus is the most close to Vasiliev's Imaginary logic. It includes standard negation $(\neg)$ and conjunction $(\&)$ together with their non-classical analogs - and $\cdot$. In contrast to classical connectives, non-classical negation $\left(^{-}\right)$can be applied only to propositional variables, and non-classical conjunction $(\cdot)$ concatenates a variable and its negation: $\bar{\gamma}$ and $\gamma \cdot \bar{\gamma}$ are the formulas if $\gamma$ is a propositional variable. In the system $\mathbf{V} 3$ formulas $\gamma, \bar{\gamma}, \gamma \cdot \bar{\gamma}$ are pairwise incompatible, and the law of excluded forth $\gamma \vee \bar{\gamma} \vee \gamma \cdot \bar{\gamma}$ is valid.

However, V3 can not be regarded as an adequate formalization of Vasiliev's Imaginary logic. The language of propositional logic is too poor to solve this problem. Imaginary logic was formulated 
by Vasiliev as a syllogistic of special kind, and the law of excluded forth is valid here only for singular propositions.

Vasiliev himself singled out the following types of basic propositions in Imaginary logic ( $v$ is an arbitrary singular term, $S$ and $P$ are any universal terms):

(1) singular:

' $v$ is $P$ ' (we'll use for them symbolic notation $\mathbf{J}_{\mathbf{1}} v P$ ),

' $v$ is not $P$ ' $-\mathbf{J}_{\mathbf{2}} v P$,

' $v$ is and is not $P$ ' $-\mathbf{J}_{\mathbf{3}} v P$;

(2) universal:

'Every $S$ is $P$ ' $-\mathbf{A}_{\mathbf{1}} S P$,

'Every $S$ is not $P$ ' $-\mathbf{A}_{2} S P$,

'Every $S$ is and is not $P$ ' $-\mathbf{A}_{\mathbf{3}} S P$;

(3) definite particular:

'Some $S$ are $P$, and all the rest of $S$ are not $P$ ' $-\mathbf{T}_{\mathbf{1}} S P$,

'Some $S$ are $P$, and all the rest of $S$ are and are not $P$ ' $-\mathbf{T}_{\mathbf{2}} S P$,

'Some $S$ are not $P$, and all the rest of $S$ are and are not $P$ ' $-\mathbf{T}_{\mathbf{3}} S P$, 'Some $S$ are $P$, some $S$ are not $P$, and all the rest of $S$ are and are not $P^{\prime}-\mathbf{T}_{4} S P$.

In addition Vasiliev used indefinite particular propositions:

'Some $S$ are $P$ ' $-\mathbf{I}_{\mathbf{1}} S P$,

'Some $S$ are not $P$ ' $-\mathbf{I}_{\mathbf{2}} S P$,

'Some $S$ are and are not $P$ ' $-\mathbf{I}_{\mathbf{3}} S P$.

T. Kostyuk and V. Markin [3] constructed the calculus IL with initial constants $\mathbf{J}_{\mathbf{1}}, \mathbf{J}_{\mathbf{2}}, \mathbf{J}_{\mathbf{3}}, \mathbf{I}_{\mathbf{1}}, \mathbf{I}_{\mathbf{2}}, \mathbf{I}_{\mathbf{3}}$ that is an adequate formalization of Vasiliev's Imaginary logic. IL contains the following deductive postulates.

Axiom schemes:

A0. Propositional tautologies,

A1. $\neg\left(\mathbf{J}_{\mathbf{1}} v P \& \mathbf{J}_{\mathbf{2}} v P\right)$,

A5. $\left(\mathbf{J}_{\mathbf{1}} v P \& \mathbf{J}_{\mathbf{1}} v S\right) \supset \mathbf{I}_{\mathbf{1}} S P$,

A2. $\neg\left(\mathbf{J}_{\mathbf{1}} v P \& \mathbf{J}_{\mathbf{3}} v P\right)$,

A6. $\left(\mathbf{J}_{\mathbf{2}} v P \& \mathbf{J}_{\mathbf{1}} v S\right) \supset \mathbf{I}_{\mathbf{2}} S P$,

A3. $\neg\left(\mathbf{J}_{\mathbf{2}} v P \& \mathbf{J}_{\mathbf{3}} v P\right)$,

A7. $\left(\mathbf{J}_{\mathbf{3}} v P \& \mathbf{J}_{\mathbf{1}} v S\right) \supset \mathbf{I}_{\mathbf{3}} S P$,

A4. $\mathbf{J}_{1} v P \vee \mathbf{J}_{\mathbf{2}} v P \vee \mathbf{J}_{\mathbf{3}} v P$,

A8. $\mathbf{I}_{1} S S$. 
Rules:
R1. $\frac{A \supset B, A}{B}$,
R3. $\frac{\left(\mathbf{J}_{\mathbf{1}} v S \& \mathbf{J}_{\mathbf{2}} v P\right) \supset A}{\mathbf{I}_{\mathbf{2}} S P \supset A}$,
R2. $\frac{\left(\mathbf{J}_{\mathbf{1}} v S \& \mathbf{J}_{\mathbf{1}} v P\right) \supset A}{\mathbf{I}_{\mathbf{1}} S P \supset A}$,
R4. $\frac{\left(\mathbf{J}_{\mathbf{1}} v S \& \mathbf{J}_{\mathbf{3}} v P\right) \supset A}{\mathbf{I}_{\mathbf{3}} S P \supset A}$

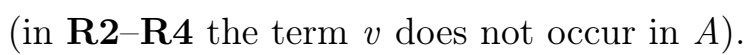

Definitions of universal and definite particular propositions:

$\mathbf{A}_{\mathbf{1}} S P \rightleftharpoons \neg \mathbf{I}_{\mathbf{2}} S P \& \neg \mathbf{I}_{\mathbf{3}} S P$,

$\mathbf{A}_{\mathbf{2}} S P \rightleftharpoons \neg \mathbf{I}_{1} S P \& \neg \mathbf{I}_{3} S P$,

$\mathbf{A}_{\mathbf{3}} S P \rightleftharpoons \neg \mathbf{I}_{1} S P \& \neg \mathbf{I}_{2} S P$,

$\mathbf{T}_{\mathbf{1}} S P \rightleftharpoons \mathbf{I}_{\mathbf{1}} S P \& \mathbf{I}_{\mathbf{2}} S P \& \neg \mathbf{I}_{\mathbf{3}} S P$,

$\mathbf{T}_{\mathbf{2}} S P \rightleftharpoons \mathbf{I}_{\mathbf{1}} S P \& \neg \mathbf{I}_{\mathbf{2}} S P \& \mathbf{I}_{\mathbf{3}} S P$,

$\mathbf{T}_{\mathbf{3}} S P \rightleftharpoons \neg \mathbf{I}_{\mathbf{1}} S P \& \mathbf{I}_{\mathbf{2}} S P \& \mathbf{I}_{\mathbf{3}} S P$,

$\mathbf{T}_{\mathbf{4}} S P \rightleftharpoons \mathbf{I}_{\mathbf{1}} S P \& \mathbf{I}_{\mathbf{2}} S P \& \mathbf{I}_{\mathbf{3}} S P$.

Formal counterparts of all the laws of Imaginary logic which Vasiliev marked out are provable in IL.

The semantics of IL proposed by T. Kostyuk and V. Markin [3] is based on assignment several extensional characteristics to each universal term - its extension, anti-extension and contradictory domain. Such an idea was implicitly presented in Vasiliev's text.

Define IL-model as follows: $\left\langle\mathbf{D}, \varphi, \psi_{1}, \psi_{2}, \psi_{3}>\right.$, where $\mathbf{D} \neq \emptyset$, $\varphi(v) \in \mathbf{D}, \psi_{1}, \psi_{2}, \psi_{3}$ are functions which put in correspondence every universal term $P$ with a subset of $\mathbf{D}$ and satisfy the following conditions: $\psi_{1}(P) \neq \emptyset, \psi_{1}(P) \cap \psi_{2}(P)=\emptyset, \psi_{1}(P) \cap \psi_{3}(P)=$ $\emptyset, \psi_{2}(P) \cap \psi_{3}(P)=\emptyset, \psi_{1}(P) \cup \psi_{2}(P) \cup \psi_{3}(P)=\mathbf{D}$.

Informally, $\psi_{1}(P)$ is an extension of $P, \psi_{2}(P)$ is an anti-extension of $P$, and $\psi_{3}(P)$ is a contradictory domain with respect to $P$.

Truth definitions for atomic formulas in a model $<\mathbf{D}, \varphi, \psi_{1}, \psi_{2}, \psi_{3}>$ :

$\left|\mathbf{J}_{\mathbf{1}} v P\right|=\mathbf{1}$ iff $\varphi(v) \in \psi_{1}(P)$,

$\left|\mathbf{J}_{\mathbf{2}} v P\right|=\mathbf{1}$ iff $\varphi(v) \in \psi_{2}(P)$,

$\left|\mathbf{J}_{\mathbf{3}} v P\right|=\mathbf{1}$ iff $\varphi(v) \in \psi_{3}(P)$,

$\left|\mathbf{I}_{\mathbf{1}} S P\right|=\mathbf{1}$ iff $\psi_{1}(S) \cap \psi_{1}(P) \neq \emptyset$,

$\left|\mathbf{I}_{2} S P\right|=\mathbf{1}$ iff $\psi_{1}(S) \cap \psi_{2}(P) \neq \emptyset$,

$\left|\mathbf{I}_{3} S P\right|=\mathbf{1}$ iff $\psi_{1}(S) \cap \psi_{3}(P) \neq \emptyset$. 
Truth definitions for complex formulas are usual.

It can easily be shown that the truth conditions for the forms of universal propositions are the following:

$$
\begin{aligned}
& \left|\mathbf{A}_{\mathbf{1}} S P\right|=\mathbf{1} \text { iff } \psi_{1}(S) \subseteq \psi_{1}(P), \\
& \left|\mathbf{A}_{2} S P\right|=\mathbf{1} \text { iff } \psi_{1}(S) \subseteq \psi_{2}(P), \\
& \left|\mathbf{A}_{3} S P\right|=\mathbf{1} \text { iff } \psi_{1}(S) \subseteq \psi_{3}(P) .
\end{aligned}
$$

A formula $A$ is true in a model $<\mathbf{D}, \varphi, \psi_{1}, \psi_{2}, \psi_{3}>$ iff $|A|=\mathbf{1}$ in this model. A formula $A$ is valid if $f$ it is true in every model.

The adequacy of the semantics for IL was proved by T. Kostyuk in her Ph.D. thesis 'Reconstruction of N.A. Vasiliev's logical systems by means of modern logic' defended in Lomonosov Moscow State University in 1999.

\section{Logic of $n$ dimensions and $n$-valued logic}

Some researchers (L. Chwistek, A.N. Maltsev, G.N. Kline, N. Rescher, M. Jammer, V.V. Anosova) considered Vasiliev to be a predecessor of many-valued logic. It appears that such an opinion is grounded on the three types of propositions' quality in his Imaginary logic. Moreover, in the paper 'Imaginary (non-Aristotelian) logic' Vasiliev advanced an idea of possible development of the logic of $n$ dimensions [1, pp. 76-77]. For him, such systems differ in a number of types of propositions varying in quality. Aristotelian syllogistic is bidimensional, imaginary logic has three dimensions. In general, a logic of $n$ dimensions must contain $n$ types of propositions with different qualities. Vasiliev himself did not develop these idea into a logical theory.

The reconstruction of the logic on $n$ dimensions was realized by T. Kostyuk [2]. She formulated an exact and intuitively transparent semantics for syllogistic language with $n$ types of propositions varying in quality along with the adequate axiomatization.

The system IL can be in a natural way extended to syllogistics $\mathbf{I L}_{\mathbf{n}}$ with arbitrary number of propositions with different qualities.

There are $n$ syllogistic constants for singular $\left(\mathbf{J}_{\mathbf{1}}, \mathbf{J}_{\mathbf{2}}, \ldots, \mathbf{J}_{\mathbf{n}}\right)$, universal $\left(\mathbf{A}_{\mathbf{1}}, \mathbf{A}_{\mathbf{2}}, \ldots, \mathbf{A}_{\mathbf{n}}\right)$ and indefinite particular $\left(\mathbf{I}_{\mathbf{1}}, \mathbf{I}_{\mathbf{2}}, \ldots, \mathbf{I}_{\mathbf{n}}\right)$ propositions of different quality. Let $\mathbf{J}_{\mathbf{i}} v P$ means that an individual $v$ stands in $i$-th qualitative relation to $P, \mathbf{A}_{1} S P$ - every object from $S$ stands in $i$-th qualitative relation to $P, \mathbf{I}_{\mathbf{1}} S P$ - some object 
from $S$ stands in $i$-th qualitative relation to $P$. When $i=1$ we have a form of affirmative proposition with corresponding quantity. It is convenient to suppose the formulas with $i=n$ to be the forms of negative propositions.

$\mathbf{I L}_{\mathbf{n}}$-model is a structure $<\mathbf{D}, \varphi, \psi_{1}, \psi_{2}, \ldots, \psi_{n}>$, where $\mathbf{D} \neq \emptyset$, $\varphi(v) \in \mathbf{D}, \psi_{i}(P) \subseteq \mathbf{D}, \psi_{1}(P) \neq \emptyset, \psi_{i}(P) \cap \psi_{j}(P)=\emptyset$, where $1 \leq i, j \leq n$ and $i \neq j ; \psi_{1}(P) \cup \psi_{2}(P) \cup \ldots \cup \psi_{n}(P)=\mathbf{D}$. In this semantical framework each universal term is connected with $n$ extensional characteristics.

The truth definitions for atomic formulas are the following:

$\left|\mathbf{J}_{\mathbf{i}} v P\right|=\mathbf{1}$ iff $\varphi(v) \in \psi_{i}(P)$,

$\left|\mathbf{A}_{\mathbf{i}} S P\right|=\mathbf{1}$ iff $\psi_{1}(S) \subseteq \psi_{i}(P)$,

$\left|\mathbf{I}_{\mathbf{i}} S P\right|=\mathbf{1}$ iff $\psi_{1}(S) \cap \psi_{i}(P) \neq \emptyset$.

Truth definitions for complex formulas are usual.

A formula $A$ is true in a model $<\mathbf{D}, \varphi, \psi_{1}, \psi_{2}, \ldots, \psi_{n}>$ iff $|A|=\mathbf{1}$ in this model. A formula $A$ is valid iff it is true in every model.

The set of $\mathbf{I L}_{\mathbf{n}}$-valid formulas is axiomatized by the calculus $\mathbf{I L}_{\mathbf{n}}$ with initial syllogistic constants $\mathbf{J}_{\mathbf{1}}, \mathbf{J}_{\mathbf{2}}, \ldots, \mathbf{J}_{\mathbf{n}}, \mathbf{I}_{\mathbf{1}}, \mathbf{I}_{\mathbf{2}}, \ldots, \mathbf{I}_{\mathbf{n}}$. Universal propositions can be defined as follows:

$\mathbf{A}_{\mathbf{i}} S P \rightleftharpoons \&_{\mathbf{j} \neq \mathbf{i}} \neg \mathbf{I}_{\mathbf{j}} S P$.

There are the following deductive postulates in $\mathbf{I L}_{\mathbf{n}}$ :

A0. Propositional tautologies,

A1. $\neg\left(\mathbf{J}_{\mathbf{i}} v P \& \mathbf{J}_{\mathbf{j}} v P\right)$, where $i \neq j$,

A2. $\mathbf{J}_{1} v P \vee \mathbf{J}_{\mathbf{2}} v P \vee \ldots \vee \mathbf{J}_{\mathbf{n}} v P$,

A3. $\left(\mathbf{J}_{\mathbf{i}} v P \& \mathbf{J}_{\mathbf{1}} v S\right) \supset \mathbf{I}_{\mathbf{i}} S P$,

A4. $\mathbf{I}_{1} S S$,

R1. $\frac{A \supset B, A}{B}$,

R2. $\frac{\left(\mathbf{J}_{1} v S \& \mathbf{J}_{\mathbf{i}} v P\right) \supset A}{\mathbf{I}_{\mathbf{i}} S P \supset A}(v$ does not occur in $A)$.

The semantical adequacy for $\mathbf{I L}_{\mathbf{n}}$ was proved by T. Kostyuk [2].

System IL turned to be three-dimensional case of manydimensional logic, while a two-dimensional case is presented by the system of traditional syllogistic with singular terms [6] that is the extension of well-known Łukasiewicz' syllogistic. 
It should be mentioned that the appearance of a proposition of a new quality does not support by itself the revision of principle of two-valuedness. Vasiliev did not consider the possibility of the third value seriously. He preferred to operate with classical valuations 'true' and 'false'.

In what follows we consider the issue of connection between Vasiliev's logical legacy and many-valuedness in a different manner - as a problem of metalogical relationship between logic of $n$ dimensions and many-valued logic.

In [5] we proposed an intuitively clear and simple adequate translation of Imaginary logic (IL calculus) into the quantified threevalued logic and proved that this translation is an embedding.

This result was generalized to an arbitrary logic of $n$ dimensions by Igor Alexeev in his graduation thesis 'Vasiliev's logic of $n$ dimensions and many-valued predicate logic', prepared at the Department of Logic, Lomonosov Moscow State University in 2009.

He has showed that axiomatic calculus $\mathbf{I L}_{\mathbf{n}}$, formalizing logic of $n$ dimensions, is embedded into monadic $n$-valued predicate logic with the following properties:

(1) $\mathbf{j}$-operators are expressible for any possible value;

(2) standard propositional connectives take the same values for classical arguments $(\mathbf{1}, \mathbf{0})$ as in classical logic;

(3) formulas of the type $\forall \alpha A$ take the value 1 iff for arbitrary value of $\alpha$, the value of $A$ is $\mathbf{1}$, and take the value $\mathbf{0}$ iff for some value of $\alpha, A$ takes the value $\mathbf{0}$;

(4) formulas of the type $\exists \alpha A$ take the value $\mathbf{1}$ iff for some value of $\alpha, A$ takes the value $\mathbf{1}$, and take the value $\mathbf{0}$ iff for arbitrary value of $\alpha$, the value of $A$ is $\mathbf{0}$.

An obvious example of such a system is quantified $n$-valued Łukasiewicz' logic $\mathrm{E}_{\mathbf{n}}$.

$\mathrm{E}_{\mathbf{n}}$-model is a structure $\left\langle\mathbf{D}, \varphi, \psi_{1}, \psi_{2}, \ldots, \psi_{n}\right\rangle$, where $\mathbf{D} \neq \emptyset$, $\varphi(v) \in \mathbf{D}, \psi_{1}, \psi_{2}, \ldots, \psi_{n}$ are the functions which put in correspondence every predicate symbol $P$ with a subset of $\mathbf{D}$ and satisfy the 
following conditions: $\psi_{i}(P) \cap \psi_{j}(P)=\emptyset$, for any $i \neq j$ from 1 up to $n ; \psi_{1}(P) \cup \psi_{2}(P) \cup \ldots \cup \psi_{n}(P)=\mathbf{D}$.

Let $\mathbf{g}$ be an assignment for variables: $\mathbf{g}(\alpha) \in \mathbf{D}$ for arbitrary variable $\alpha$.

The set of possible values for formulas is $\left\{\mathbf{1}, \frac{\mathbf{n}-\mathbf{2}}{\mathbf{n}-\mathbf{1}}, \ldots, \frac{\mathbf{1}}{\mathbf{n}-\mathbf{1}}, \mathbf{0}\right\}$.

Valuation for terms and formulas is defined as follows:

$\mathbf{V}_{\mathbf{g}}(\alpha)=\mathbf{g}(\alpha), \quad \mathbf{V}_{\mathbf{g}}(v)=\varphi(v)$,

$\mathbf{V}_{\mathbf{g}}(P t)=\frac{\mathbf{n}-\mathbf{i}}{\mathbf{n}-\mathbf{1}}$ iff $\mathbf{V}_{\mathbf{g}}(t) \in \psi_{i}(P)$,

$\mathbf{V}_{\mathbf{g}}(\neg A)=\mathbf{1}-\mathbf{V}_{\mathbf{g}}(A)$,

$\mathbf{V}_{\mathbf{g}}(A \& B)=\min \left(\mathbf{V}_{\mathbf{g}}(A), \mathbf{V}_{\mathbf{g}}(B)\right)$,

$\mathbf{V}_{\mathbf{g}}(A \vee B)=\max \left(\mathbf{V}_{\mathbf{g}}(A), \mathbf{V}_{\mathbf{g}}(B)\right)$,

$\mathbf{V}_{\mathbf{g}}(A \supset B)=\min \left(\mathbf{1}, \mathbf{1}-\mathbf{V}_{\mathbf{g}}(A)+\mathbf{V}_{\mathbf{g}}(B)\right)$,

$\mathbf{V}_{\mathbf{g}}(\forall \alpha A)=\frac{\mathbf{n}-\mathbf{i}}{\mathbf{n}-\mathbf{1}}$ iff $\quad \mathbf{V}_{\mathbf{g}^{\prime}}(A)=\frac{\mathbf{n}-\mathbf{i}}{\mathbf{n}-\mathbf{1}}$ for some $\mathbf{g}^{\prime}={ }_{\alpha} \mathbf{g}$, and there is no $\mathbf{g}^{\prime}={ }_{\alpha} \mathbf{g}$ such that $\mathbf{V}_{\mathbf{g}^{\prime}}(A)<\frac{\mathbf{n}-\mathbf{i}}{\mathbf{n}-\mathbf{1}}$,

$\mathbf{V}_{\mathbf{g}}(\exists \alpha A)=\frac{\mathbf{n}-\mathbf{i}}{\mathbf{n}-\mathbf{1}}$ iff $\quad \mathbf{V}_{\mathbf{g}^{\prime}}(A)=\frac{\mathbf{n}-\mathbf{i}}{\mathbf{n}-\mathbf{1}}$ for some $\mathbf{g}^{\prime}={ }_{\alpha} \mathbf{g}$, and there is no $\mathbf{g}^{\prime}={ }_{\alpha} \mathbf{g}$ such that $\mathbf{V}_{\mathbf{g}^{\prime}}(A)>\frac{\mathbf{n}-\mathbf{i}}{\mathbf{n}-\mathbf{1}}$

$\left(\mathbf{g}^{\prime}={ }_{\alpha} \mathbf{g}\right.$ means the following: $\mathbf{g}^{\prime}$ differs from $\mathbf{g}$ at most in assignment for $\alpha$ ).

$\mathbf{j}$-operators are expressible in $\mathrm{E}_{\mathbf{n}}$ by the following interpretation:

$\mathbf{V}_{\mathbf{g}}\left(\mathbf{j}_{\mathbf{i}} A\right)=\mathbf{1}$, if $\mathbf{V}_{\mathbf{g}}(A)=\frac{\mathbf{n}-\mathbf{i}}{\mathbf{n}-\mathbf{1}}$, otherwise $-\mathbf{V}_{\mathbf{g}}\left(\mathbf{j}_{\mathbf{i}} A\right)=\mathbf{0}$.

Formula $A$ is valid in $\mathbf{E}_{\mathbf{n}}$-model iff $\mathbf{V}_{\mathbf{g}}(A)=\mathbf{1}$, for any assignment g. Formula $A$ is valid iff $A$ is valid in any $\mathrm{七}_{\mathbf{n}}$-model.

The embedding operation from logic of $n$ dimensions $\mathbf{I L}_{\mathbf{n}}$ into quantified $n$-valued logic $\mathrm{E}_{\mathbf{n}}$ is defined in two stages.

First define the mapping ${ }^{\star}$ of the set of $\mathbf{I L}_{\mathbf{n}}$-formulas into the set of formulas of quantified $n$-valued Łukasiewicz' logic:

$\left(\mathbf{J}_{\mathbf{i}} v P\right)^{\star}=\mathbf{j}_{\mathbf{i}} P v$,

$\left(\mathbf{I}_{\mathbf{i}} S P\right)^{\star}=\exists x\left(\mathbf{j}_{1} S x \& \mathbf{j}_{\mathbf{i}} P x\right)$,

$(\neg A)^{\star}=\neg A^{\star}$,

$(A \otimes B)^{\star}=A^{\star} \otimes B^{\star}$, where $\otimes$ is any binary connective.

Further on the basis of ${ }^{\star}$ define the embedding operation $\Theta$ :

$$
\Theta(A)=\left(\exists x \mathbf{j}_{1} S_{1} x \& \exists x \mathbf{j}_{1} S_{2} x \& \ldots \& \exists x \mathbf{j}_{1} S_{m} x\right) \supset A^{\star}
$$


where $A$ is an arbitrary formula of $\mathbf{I L}_{\mathbf{n}}$ language, and $S_{1}, S_{2}, \ldots, S_{m}$ is the list of all universal terms in $A$.

This result shows the existence of a natural interpretation of any Vasiliev's $n$-dimensional logic (including his Imaginary logic) in a quantified many-valued logic's framework.

\section{Imaginary logic as intensional logic}

In the final part of the paper 'Imaginary (non-Aristotelian) logic' Vasiliev made an attempt to formulate intensional semantics for the propositions of his logical system 'Every $S$ is $P$ ', 'Every $S$ is not $P$ ', 'Every $S$ is and is not $P$ '.

Vasiliev compares Imaginary logic with non-Euclidian geometry and poses a question about possible interpretation of Imaginary logic in terms of our terrestrial world:

'We can propose a real interpretation of Non-Euclidian geometry, we can find in our Euclidian space the essences with non-Euclidean geometry... A real interpretation of Lobachevsky's geometry is a geometry of surface with constant negative curvature, of so called pseudosphere... In exactly the same way it is possible to find in our world the essences with the logic analogous to imaginary logic' [1, p. 81].

Vasiliev proposed three 'terrestrial' interpretations of Imaginary logic. The core idea of the most interesting interpretation is to associate with each term of a categorical statement not a set of individuals but a concept considered as a set of characters and to treat syllogistic constants as denoting intensional relations between concepts. According to this approach, 'Every $S$ is $P$ ' means that $S$ contains all characters from $P$. 'Every $S$ is not $P$ ' means that, for an arbitrary character from $P$, the concept $S$ contains contradictory one, 'Every $S$ is and is not $P$ ' means that $S$ contains some characters from $P$ as well as characters which contradict to some others.

Vasiliev emphasized that the logic of concepts based on such semantics differs from the main version of Imaginary logic as well as from the standard syllogistic. For example, some first figure syllo- 
gisms with minor negative premise are valid: 'Every $M$ is $P$. Every $S$ is not $M$. Hence, every $S$ is not $P^{\prime}$.

Vasiliev's ideas, related to this fragment of his paper, were explicated semantically by V. Markin and D. Zaitsev [8].

Let $\mathbf{L}$ be a set of literals - positive and negative characters $\left\{p_{1}, \sim p_{1}, p_{2}, \sim p_{2}, \ldots\right\}$.

Then a concept is an arbitrary non-empty and consistent subset of $\mathbf{L}$, i.e. a set $\alpha \subseteq \mathbf{L}$, which satisfies the following conditions:

(i) $\alpha \neq \emptyset ; \quad$ (ii) there is no $p_{i}: p_{i} \in \alpha$ and $\sim p_{i} \in \alpha$.

Let $\mathbf{M}$ be the set of all concepts. We define an operation * on $\mathbf{M}$, which assigns to every concept $\alpha$ a contrary concept $\alpha^{*}$ :

$$
p_{i} \in \alpha^{*} \text { iff } \sim p_{i} \in \alpha \text { and } \sim p_{i} \in \alpha^{*} \text { iff } p_{i} \in \alpha .
$$

Vasiliev himself used the same operation:

'If the concept $A$ consists of characters $p, q, r, s, \ldots$ then the concept non- $A$ must consist of characters non- $p$, non$q$, non- $r$, non-s, and so on' [1, p. 88].

Vasiliev proposed semantical definitions only for universal statements. As before, let $\mathbf{A}_{\mathbf{1}} S P$ be the form of universal affirmative statements 'Every $S$ is $P$ ', $\mathbf{A}_{\mathbf{2}} S P$ - the form of universal negative statements 'Every $S$ is not $P$ ', and $\mathbf{A}_{\mathbf{3}} S P$ - the form of universal indifferent statements 'Every $S$ is and is not $P$ '.

Let $\mathbf{d}$ be a function assigning arbitrary concepts to terms: $\mathbf{d}(P) \in \mathbf{M}$. Define a valuation associated with $\mathbf{d}$ :

$$
\begin{aligned}
& \left|\mathbf{A}_{\mathbf{1}} S P\right|^{\mathbf{d}}=\mathbf{1} \text { iff } \mathbf{d}(P) \subseteq \mathbf{d}(S), \\
& \left|\mathbf{A}_{\mathbf{2}} S P\right|^{\mathbf{d}}=\mathbf{1} \text { iff } \mathbf{d}(P)^{*} \subseteq \mathbf{d}(S), \\
& \left|\mathbf{A}_{\mathbf{3}} S P\right|^{\mathbf{d}}=\mathbf{1} \text { iff } \mathbf{d}(P) \cap \mathbf{d}(S) \neq \emptyset \text { and } \mathbf{d}(P)^{*} \cap \mathbf{d}(S) \neq \emptyset .
\end{aligned}
$$

However to formulate complete system of Imaginary logic one needs more then just universal statements. In the main version of this logic Vasiliev uses as well particular statements: 'Some $S$ are $P^{\prime}\left(\mathbf{I}_{\mathbf{1}} S P\right)$, 'Some $S$ are not $P^{\prime}\left(\mathbf{I}_{\mathbf{2}} S P\right)$ and 'Some $S$ are and are not $P^{\prime}\left(\mathbf{I}_{\mathbf{3}} S P\right)$. V. Markin and D. Zaitsev [8] offer the following truth definitions for the particular propositions:

$\left|\mathbf{I}_{\mathbf{1}} S P\right|^{\mathbf{d}}=\mathbf{1}$ iff $\mathbf{d}(P)^{*} \cap \mathbf{d}(S)=\emptyset$, 


$$
\left\{\begin{array}{l}
\left.\mathbf{I}_{\mathbf{2}} S P\right|^{\mathbf{d}}=\mathbf{1} \text { iff } \mathbf{d}(P) \cap \mathbf{d}(S)=\emptyset \\
\left|\mathbf{I}_{\mathbf{3}} S P\right|^{\mathbf{d}=\mathbf{1}} \text { iff } \mathbf{d}(P) \backslash \mathbf{d}(S) \neq \emptyset \text { and } \mathbf{d}(P)^{*} \backslash \mathbf{d}(S) \neq \emptyset,
\end{array}\right.
$$

and usual truth definitions for complex formulas.

A formula is valid in this 'intensional' semantics iff it takes value ' $\mathbf{1}$ ' under any assignment $\mathbf{d}$.

The set of valid formulas is axiomatized by the calculus IL2 containing propositional tautologies and axiom schemes:

A1. $\left(\mathbf{A}_{\mathbf{1}} M P \& \mathbf{A}_{\mathbf{1}} S M\right) \supset \mathbf{A}_{\mathbf{1}} S P, \quad \mathbf{A} 10 . \neg\left(\mathbf{A}_{\mathbf{1}} S P \& \mathbf{I}_{\mathbf{2}} S P\right)$,

A2. $\left(\mathbf{A}_{\mathbf{1}} M P \& \mathbf{A}_{\mathbf{2}} S M\right) \supset \mathbf{A}_{\mathbf{2}} S P, \quad \mathbf{A 1 1} . \neg\left(\mathbf{A}_{\mathbf{2}} S P \& \mathbf{I}_{\mathbf{1}} S P\right)$,

A3. $\left(\mathbf{A}_{\mathbf{2}} M P \& \mathbf{A}_{\mathbf{1}} S M\right) \supset \mathbf{A}_{\mathbf{2}} S P, \quad \mathbf{A} 12 . \mathbf{I}_{1} S P \supset \mathbf{I}_{\mathbf{1}} P S$,

A4. $\left(\mathbf{A}_{\mathbf{2}} M P \& \mathbf{A}_{\mathbf{2}} S M\right) \supset \mathbf{A}_{\mathbf{1}} S P, \mathbf{A 1 3} . \mathbf{I}_{\mathbf{2}} S P \supset \mathbf{I}_{\mathbf{2}} P S$,

A5. $\left(\mathbf{A}_{1} M P \& \mathbf{I}_{1} S M\right) \supset \mathbf{I}_{1} S P, \quad \mathbf{A} 14 . \mathbf{A}_{1} S P \supset \mathbf{I}_{1} S P$,

A6. $\left(\mathbf{A}_{1} M P \& \mathbf{I}_{2} S M\right) \supset \mathbf{I}_{\mathbf{2}} S P, \quad$ A15. $\mathbf{A}_{\mathbf{2}} S P \supset \mathbf{I}_{\mathbf{2}} S P$,

A7. $\left(\mathbf{A}_{\mathbf{2}} M P \& \mathbf{I}_{\mathbf{1}} S M\right) \supset \mathbf{I}_{\mathbf{2}} S P, \quad$ A16. $\mathbf{A}_{\mathbf{3}} S P \equiv \neg \mathbf{I}_{\mathbf{1}} S P \& \neg \mathbf{I}_{\mathbf{2}} S P$,

A8. $\left(\mathbf{A}_{\mathbf{2}} M P \& \mathbf{I}_{2} S M\right) \supset \mathbf{I}_{1} S P, \quad \mathbf{A} 17 . \mathbf{I}_{3} S P \equiv \neg \mathbf{A}_{\mathbf{1}} S P \& \neg \mathbf{A}_{\mathbf{2}} S P$.

A9. $\mathbf{A}_{1} S S$,

The only rule is modus ponens.

Thus, Vasiliev has developed a sketch of the alternative version of Imaginary logic based on intensional interpretation of its propositions. He showed the manifold of non-classical logical systems, which are formulated in the same language and differ from each other in sets of laws.

\section{Some ideas of modal and temporal logics}

In his first paper 'On particular statements, the triangle of oppositions, the law of excluded forth' Vasiliev proposed to treat singular statements as temporal i.e. containing either temporal parameter or temporal characteristic. Vasiliev differentiates two kinds of singular statements: 'statements on the fact' and 'statements on the concept'.

Singular statements on the fact refer to an individual in the certain moment in time, to definite state of the individual in the history of its existence:

'The copula of such statements presumes the exact designation of temporal moment, for the subjects of such 
singular statements - perceptions and mental representations - always refer to the certain moment of time' $[1$, p. 51].

Vasiliev gives the following examples of the statements on the fact: 'Ivan Ivanovich is drunk now', ' $N N$ passed away at 5 a.m. yesterday', ' $N N$ is sick today'.

The subject of the statements on the concept represents the set of all possible states of an individual over the time of its existence:

'The subject of singular statement Caesar, Goethe etc. can be a concept, and then it symbolizes all terrestrial life of Caesar and Goethe, it subordinates the set of certain moments of Caesar's and Goethe's life to the unity of the concept' [1, p. 51];

'All these certain moments in Caesar's life: Caesar with the robbers, Caesar as the conqueror of Vercingetorix, Caesar as the monarch, Caesar as the lover of Cleopatra, Caesar killed with conspirators' dagger, - all of them are symbolized in the united concept 'Caesar' in the same way as Caesar, Pompeius and Gaius are symbolized in the united concept of human being' [1, p. 50].

Then there are three kinds of the statements on the concept: (1) an individual always has a property, (2) an individual never has a property, (3) an individual sometimes has a property, and sometimes has not:

'Indeed, the predicate of humanity is appropriable to Caesar in every moment of his existence, the predicate of triangularity is not appropriable at all, and the predicate of sickness is appropriable to some moments, and is not appropriable to others' $[1$, p. 50$]$.

Vasiliev stresses that singular statements on the fact and singular statements on the concept obey different logical laws. The law of excluded middle is valid for the statements on the fact. For example, only one of two statements 'Ivan Ivanovich is drunk now' and 'Ivan Ivanovich is not drunk now' is true. Statements on the concept 
obey another law - the law of excluded forth. For example, one of three statements 'Vega always shines', 'Vega never shines', 'Vega sometimes shines, and sometimes does not' is true, and these three propositions are pairwise incompatible.

Vasiliev also supposes any singular statement on the concept to express the certain rule:

'They describe time series as a rule, and the basic law for rules, the law of excluded forth acts for them' $[1$, p. 51].

Thereby, Vasiliev in fact treats these statements as modal.

Vasiliev also considers the modal treatment of the propositions in his main paper 'Imaginary (non-Aristotelian) logic'. Here he studies statements with universal, but not singular subjects.

In this paper Vasiliev proposed not only intensional but also modal interpretation of categorical statements containing in Imaginary logic:

'If we take a concept as a subject of a statement, then any predicate refers to it either as 1) this predicate is necessary for the concept..., and we express this fact in affirmative statement about the concept..., or as 2) this predicate is impossible for the concept..., and we express this fact in negative statement about the concept..., or as 3) this predicate is compatible with the concept... The third case should be expressed in special accidental statement about the concept... This statement has its special copula different from the copulae of affirmative and negative statements' [1, pp. 81-82].

The character of this copula is clear from another fragment where Vasiliev specifies the form of the accidental statements: ' $S$ possibly is and possibly is not $P^{\prime}[1$, p. 125].

Obviously, these modalities used by Vasiliev describe the type of predication, the mode of connection between the subject and the predicate, i.e. they are de re modalities.

Affirmative propositions are treated as containing modality of necessary inherence of a property to an individual, negative as containing modality of necessary lack (or impossibility for an individual 
to have a property), and indifferent as containing modality of contingency.

The formal explication of modal interpretation of Imaginary logic by means of special logic with de re modalities was proposed by V. Markin in [4]. The translation of formulas of the system IL into the language of logic for de re modalities was presented. It was demonstrated that the translations of all IL theorems are valid in this modal logic, while the translations of all theses rejected by Vasiliev are not valid here. Therefore, this modal interpretation is reasonable just for the main version of Imaginary logic. In contrast to 'intensional interpretation' it is not required to revise the set of its laws.

\section{References}

[1] Vasiliev, N. A., Imaginary logic. Selected works, Moscow: Science, 1989 (in Russian)

[2] Kostyuk, T. P., N. A. Vasiliev's $N$ dimensional logic: modern reconstruction, Logical investigations 7:261-268, 2000 (in Russian).

[3] Kostyuk, T. P., and V. I. Markin, Formal reconstraction of imaginary logic of N.A. Vasiliev, Modern logic: theory, history, and applications in science, proceedings of $V$ all-Russian scientific conference, St. Petersburg: Publishing House of St. Petersburg State University, 1998, pp. 154-159 (in Russian).

[4] Markin, V. I., Modal interpretation of imaginary logic of N.A. Vasiliev, Modern logic: theory, history, and applications in science, proceedings of V all-Russian scientific conference, St. Petersburg: Publishing House of St. Petersburg State University, 1998, pp. 208-214 (in Russian).

[5] Markin, V. I., Embedding of N.A. Vasiliev's imaginary logic into quantifier three-valued logic, Logical investigations 7:252-260, 2000 (in Russian).

[6] Markin, V. I., Singular extensions of Łukasiewicz syllogistic, Logical investigations 17:173-190, 2011 (in Russian).

[7] Arruda, A. I., On the imaginary logic of N.A. Vasil'ev, Proceedings of Fourth Latin-American Symposium on Mathematical Logic. NorthHolland, 1979, pp. 1-41.

[8] Markin, V. I., and D. V. Zaitsev, Imaginary logic-2: formal reconstruction of the unnoticed Nikolai Vasiliev's logical system, Logique et Analyse 45(177-178):39-54, 2002. 